Flávia Janólio Costacurta Pinto da Silva ${ }^{1}$ Paulo Sérgio Faro Santos² Francisco do Prado Reis ${ }^{3}$ Sônia Oliveira Lima ${ }^{4}$

${ }^{1}$ Mestre em Saúde e Ambiente e Professora Assistente do Departamento de Enfermagem da Universidade Federal de Sergipe, Aracaju, SE, Brasil.

${ }^{2}$ Acadêmico de Medicina da Universidade Federal de Sergipe, Aracaju, SE, Brasil.

${ }^{3}$ Professor Titular da Faculdade de Medicina da Universidade Tiradentes, Aracaju, SE, Brasil.

${ }^{4}$ Professora Adjunta Doutora do Departamento de Medicina da Universidade Federal de Sergipe; Professora Permanente do Programa de Pós-graduação em Saúde e Ambiente da Universidade Tiradentes, Aracaju, SE, Brasil.

* Trabalho baseado na dissertação de Mestrado em Saúde e Ambiente de Flávia Janólio Costacurta Pinto da Silva, intitulada Ambiente hospitalar: acidentes ocupacionais e a contaminação por hepatite $B$, apresentada ao Programa de Pós-graduação em Saúde e Ambiente da Universidade Tiradentes, em 2008.

Contato:

Sônia Oliveira Lima

Avenida Beira Mar, 1.044 - Treze de Julho, Aracaju, Sergipe, Brasil

CEP 49060-100

E-mail:

sonia.sol@ibest.com.br

\section{Estado vacinal e conhecimento dos profissionais de saúde sobre hepatite $B$ em um hospital público do nordeste brasileiro*}

\author{
Vaccination status and knowledge on hepatitis B among \\ healthcare workers of a public hospital in Northeastern Brazil
}

\section{Resumo}

Objetivo: analisar o estado vacinal e o conhecimento prévio sobre o vírus da hepatite B pelos profissionais de saúde (PS) de um hospital público. Métodos: estudo descritivo de corte transversal, baseado no preenchimento do formulário pelos PS, contemplando: biomédicos, odontólogos, enfermeiros, fisioterapeutas, médicos, técnicos e auxiliares de enfermagem e de laboratório. Para analisar idade, categoria profissional, estado vacinal e conhecimento destes profissionais sobre hepatite $\mathrm{B}$, utilizou-se o teste de qui-quadrado, considerando significante $\mathrm{p}<0,05$. Resultados: durante quatro meses de 2007, foram questionados 861 profissionais no principal hospital de urgência da rede pública de Sergipe. Houve uma relação estatisticamente significante entre grau de escolaridade e conhecimento acerca da hepatite B pelos PS. Quanto ao estado vacinal dos participantes, constatou-se que 67\% declararam estar completamente imunizados, enquanto $21,6 \%$ apresentavam-se incompletamente vacinados, $6,2 \%$ desconheciam seu estado vacinal e 5,2\% não eram vacinados. Entre os de nível superior, 75,3\% estavam completamente imunizados, comparados a $64,5 \%$ do nível técnico. Conclusão: os PS com nível superior mostraram mais conhecimento sobre o tema em detrimento dos profissionais com nível técnico, razão que deveria motivar os gestores da saúde a implantar programas de educação continuada, além de desenvolver pesquisas neste âmbito.

Palavras-chave: estado vacinal; profissionais de saúde; hepatite B.

\begin{abstract}
Objective: To analyze the vaccination status and knowledge on hepatitis $B$ virus of healthcare workers (HW) from a public hospital. Methods: Cross-sectional study, based on a written questionnaire answered by HW, which included biomedical personnel, dentists, nurses, physiotherapists, physicians, nurse assistants, and lab technicians. They informed about their age, profession, immunization status and knowledge on hepatitis B. Chi-square test was used, considering $p<0.05$ as statistically significant. Results: During four months in 2007, questionnaires were filled by 861 professionals from the main public emergency hospital in Sergipe State, Northeastern region of Brazil. There was a statistically significant relationship between educational level and $H W^{\prime} s$ previous knowledge on hepatitis B. As for the vaccination status, $67 \%$ of the HW reported being fully immunized, while $21.6 \%$ had not taken all the vaccines, $6.2 \%$ was unaware of their status, and $5.2 \%$ was not vaccinated. Among university level HW $75.3 \%$ was fully immunized, while 64,5\% of high-school-level technicians was. Conclusion: University degree HW showed a greater knowledge on the subject when compared to technicians, results that should motivate managers in healthcare to implement continuing education for their personnel as well as research about this topic.
\end{abstract}

Keywords: immunization status; healthcare workers; hepatitis B. 


\section{Introdução}

Embora os serviços de saúde, em particular as instituições hospitalares, sejam as principais fontes empregadoras dos profissionais da saúde, deve-se ressaltar que são locais propícios para a ocorrência de agravos à saúde desses profissionais, devido à constante exposição ao ambiente insalubre (SÊCCO et al., 2002).

O ambiente hospitalar é o local onde se concentram pacientes acometidos pelas mais variadas doenças e assistidos por diferentes categorias de Profissionais da Saúde (PS). Inúmeros estudos demonstram as condições impróprias de trabalho em grande parte dessas instituições de saúde, expondo os trabalhadores a riscos ocupacionais causados por fatores químicos, físicos, ergonômicos, mecânicos, psicossociais e biológicos (BARBOSA; SOLER, 2003).

Quanto ao risco biológico nas instituições de saúde, não está somente relacionado à interação do trabalhador com pacientes portadores de agentes infecciosos (vírus, bactérias, parasitas etc.), mas também a materiais contaminados com fluídos biológicos. De acordo com Bolyard et al. (1998), pode-se relacionar cerca de 20 doenças passíveis de serem transmitidas por meio desta interação. Os patógenos veiculados pelo sangue, como o vírus da imunodeficiência humana (VIH), os vírus da hepatite C (VHC) e da hepatite B (VHB), são os de maior impacto na saúde dos profissionais.

Atualmente, considera-se a hepatite B a doença infecciosa com maior probabilidade de ser adquirida pelos profissionais da saúde na execução de suas atividades laborais (AZIZ et al., 2002; CHIARAKUL et al., 2007). O risco de um profissional de saúde se contaminar com o VHB é 100 vezes maior do que o risco de contaminação pelo VIH, além de 10 vezes maior que pelo VHC (YOSHIDA, 1998). Pesquisadores alertam para o risco ainda maior dos profissionais não imunizados ou incompletamente imunizados, os quais apresentam uma probabilidade de contaminação 30 vezes superior pelo VHB quando comparados com a população em geral (BONANI; BONACCORSI, 2001; RISCHITELLI et al., 2001).

O profissional de saúde está sujeito a várias formas de exposição ao VHB no ambiente laboral. Estas vias de contaminação incluem a inoculação percutânea de sangue contaminado por meio de agulhas ou objetos cortantes, o contato com mucosas (olhos, boca, nariz ou genitália) por meio de respingos e o contato com pacientes apresentando dermatite ou feridas abertas em solução de continuidade da pele (RAPPARINI, 2007). Mas o acidente com material perfurocortante contaminado é reco- nhecidamente o maior responsável pela transmissão de hepatite $\mathrm{B}$ entre os profissionais da saúde. O risco de contaminação desta infecção está relacionado tanto ao grau de exposição ao sangue, como também à presença do antígeno de superfície AgHbe no paciente-fonte, entre outros fatores (BRASIL, 2005). Estima-se que o risco de contaminação pelo VHB após acidente com perfurocortante varie entre $6 \%$ a $30 \%$, sendo significativamente maior que o risco pelo VIH $(0,3 \%$ a $0,4 \%)$ e pelo VHC $(0,5 \%$ a $2 \%)$ (BRASIL, 1999). O risco ocupacional de exposição percutânea e mucocutânea a material biológico também está diretamente relacionado à categoria profissional, à atividade desenvolvida no ambiente laboral e ao setor de atuação do trabalhador na instituição hospitalar. De acordo com Rapparini (2007), os "profissionais da saúde da área cirúrgica, odontólogos, paramédicos e profissionais de setores de atendimento de emergência são descritos como profissionais de alto risco por serem mais expostos".

Estudos realizados na França e na Itália sobre a notificação de acidentes com exposição a material biológico mostraram-se unânimes em declarar que os acidentes perfurocortantes foram os mais prevalentes nesses países, variando apenas a sua frequência na população estudada (TARANTOLA et al., 2003; IPPOLITO; PURO; De CARLI, 1993; PERRY; PARKER; JAGGER, 2005). Nos Estados Unidos, estimativas revelam a ocorrência aproximada de 385 mil acidentes percutâneos por ano (PANILILIO et al., 2004). No Brasil, os resultados obtidos em estudos realizados por Souza (1999), Basso (1999) e Coutinho et al. (2002) não diferiram dos encontrados nos países citados anteriormente. Contudo, Canini et al. (2002) afirmaram que os dados sobre acidentes ocupacionais com material biológico não contam com uma sistematização de notificações, impossibilitando, desta forma, conhecer a magnitude do problema no Brasil.

$\mathrm{O}$ presente estudo tem por objetivo analisar o estado vacinal e o conhecimento prévio sobre o vírus da hepatite B pelos profissionais de saúde de um hospital público do estado de Sergipe, Brasil.

\section{Casuística e métodos}

Estudo descritivo de corte transversal, desenvolvido em um hospital de urgência de alta complexidade pertencente à rede pública de Sergipe, no período de maio a agosto de 2007. A instituição está situada em Aracaju e atende a população dos municípios do estado de Sergipe e dos circunvizinhos. Conta com 376 leitos distribuídos entre as especialidades cirúrgica, clínica médica, pediátrica e oncológica. Dentre os setores que compõem o hospital, foram incluídas somente as unidades de assistência direta e indireta ao paciente, 
como: unidade de Urgência/Emergência (UE), Centro Cirúrgico (CC), Central de Esterilização (CE), Unidades de Terapia Intensiva (UTI) - adulto, infantil e queimados -, Unidades de Internação (adulto e infantil).

$\mathrm{O}$ instrumento de investigação foi baseado no formulário de Ciorlia e Zanetta (2003), o qual foi adaptado para este estudo. Realizou-se teste piloto com 15 profissionais de saúde, escolhidos aleatoriamente na instituição estudada. Foram abordadas variáveis como: idade, gênero, categoria profissional, conhecimento prévio dos profissionais da saúde sobre hepatite B e estado vacinal declarado contra ela, não sendo avaliada a carteira vacinal.

Após a aprovação do Comitê de Ética e Pesquisa da Universidade Tiradentes e da assinatura do consentimento informado, os profissionais de saúde preencheram o formulário a próprio punho, no horário de trabalho, contemplando os turnos manhã, tarde e noite. Foram definidos como profissionais da saúde para este estudo aqueles listados na relação fornecida pelo Departamento de Recursos Humanos que mantêm contato direto com pacientes e manuseiam objetos utilizados por eles em ambientes de assistência. O critério de inclusão foi ser funcionário concursado, sendo excluídos da pesquisa não os profissionais com contrato de trabalho temporário, mas os concursados que estavam afastados da instituição no período da coleta.

As categorias contempladas foram biomédicos, odontólogos, enfermeiros, fisioterapeutas, médicos, técnicos e auxiliares de enfermagem e técnicos e auxiliares de laboratório. Não foi considerada distinção entre as categorias de auxiliar (aux.) e técnico (tec.) de enfermagem, como também de aux./tec. de laboratório, visto possuírem formação de nível técnico e não haver diferença de função e atividades desempenhadas na instituição estudada.

Os dados obtidos foram codificados e inseridos no programa estatístico Epi-Info versão 6.02. Utilizou-se estatística descritiva para análise das variáveis idade, gênero e categoria profissional e teste de qui-quadrado para analisar as diferenças entre as variáveis. $\mathrm{O}$ valor considerado como significante foi $\mathrm{p}<0,05$.

\section{Resultados}

Do total de 1.365 profissionais da saúde elegíveis para o estudo, $353(25,8 \%)$ se recusaram a participar da pesquisa e $151(11,1 \%)$ estavam afastados no período da coleta. Com isso, o grupo avaliado foi de 861 trabalhadores, que correspondeu a $63 \%$ dos profissionais da área da saúde da instituição.
Poucas questões do formulário não foram assinaladas, o que promoveu um percentual diferenciado de respondentes por variável, o qual será expresso com seus respectivos resultados. Referente à fonte de informação sobre hepatite B, mais de um item na questão pode ser assinalado. As respostas foram compactadas em: fonte formal (participação do funcionário em cursos, seminários específicos, universidade ou curso profissionalizante), fonte não formal (jornais/revistas, televisão/rádio, amigos/familiares), profissionais da saúde (informação de profissional capacitado) e outros.

A fonte formal de conhecimento sobre hepatite B obteve a maior frequência de respostas $(67,4 \%)$, seguida de informações transmitidas por profissionais da saúde $(49,1 \%)$ e fonte não formal $(44,1 \%)$. O número de profissionais que declarou não ter recebido nenhuma informação sobre o assunto foi de $1 \%(n=9)$. Apenas $0,1 \%(n=1)$ não respondeu à questão.

Quanto ao conhecimento prévio sobre a hepatite $\mathrm{B}$ relacionado à categoria profissional, foram consideradas como afirmativas corretas: o vírus da hepatite B pode estar presente no sangue e em fluidos corpóreos contendo ou não sangue; a hepatite B pode ser adquirida no ambiente hospitalar através do contato do profissional com sangue e fluidos corpóreos em pele e/ou mucosa lesionada; a hepatite B é uma doença de fácil contaminação na ocorrência de acidente com material biológico.

Observou-se uma relação estatisticamente significativa entre grau de escolaridade e conhecimento prévio, sendo que os profissionais de nível superior apresentaram as maiores frequências de respostas corretas (Tabela 1).

Nesta pesquisa, dos 861 respondentes sobre o estado vacinal contra hepatite $B$, verificou-se que 577 (67\%) declararam estar com o estado vacinal completo, enquanto 186 (21,6\%) apresentavam-se imunizados incompletamente (1 ou 2 doses), $53(6,2 \%)$ desconheciam seu estado vacinal e 45 (5,2\%) não eram vacinados. É notório, portanto, que $33 \%$ dos profissionais de saúde estavam susceptíveis ao VHB.

A frequência da imunização da hepatite B entre as categorias de nível técnico (aux./tec. de enfermagem e laboratório), foi de $35,5 \%$ de indivíduos não ou incompletamente imunizados contra $24,7 \%$ entre os profissionais do nível superior (médicos, enfermeiros etc.). E o estado vacinal completo dos profissionais com escolaridade de nível superior foi de $75,3 \%(p=0,005)$, dados esses que corroboram 
a pesquisa de Silva et al. (2003), que verificaram maior frequência de imunizados nas categorias de profissionais da saúde com terceiro grau completo.

Foi observada neste estudo uma correlação inversa em relação à faixa etária, ou seja, à medida que a idade aumenta, diminui significativamente o percentual de indivíduos vacinados $(\mathrm{p}=0,003)$ (Figura 1). Resultado também evidenciado no estudo de Silva et al., (2003), os quais acreditam que tal fato se deva à incorporação de informação sobre hepatites virais no ensino acadêmico e das medidas de biossegurança a partir da década de 1990.

Tabela 1 Resposta quanto ao conhecimento sobre hepatite B, segundo categoria dos profissionais de saúde de um hospital de emergências de Sergipe, 2007

\begin{tabular}{|c|c|c|c|c|c|}
\hline \multirow{2}{*}{ Variável } & \multirow{2}{*}{ Categorias } & \multicolumn{2}{|c|}{ Respostas } & \multirow{2}{*}{ Total } & \multirow{2}{*}{$P$} \\
\hline & & Certo & Errado & & \\
\hline \multirow{2}{*}{$\begin{array}{l}\text { O vírus da hepatite B pode } \\
\text { estar presente no sangue e } \\
\text { em fluidos corpóreos con- } \\
\text { tendo ou não sangue? }\end{array}$} & Técnico & $\begin{array}{c}253 \\
41,7 \%\end{array}$ & $\begin{array}{c}354 \\
58,3 \%\end{array}$ & $\begin{array}{c}607 \\
100,0 \%\end{array}$ & \multirow{2}{*}{0,023} \\
\hline & Superior & $\begin{array}{c}123 \\
50,2 \%\end{array}$ & $\begin{array}{c}122 \\
49,8 \%\end{array}$ & $\begin{array}{c}245 \\
100,0 \%\end{array}$ & \\
\hline \multirow{2}{*}{$\begin{array}{l}\text { Contato com sangue e fluídos } \\
\text { corpóreos em pele e/ou } \\
\text { mucosa lesionada }\end{array}$} & Técnico & $\begin{array}{c}78 \\
12,9 \%\end{array}$ & $\begin{array}{c}529 \\
87,1 \%\end{array}$ & $\begin{array}{c}607 \\
100,0 \%\end{array}$ & \multirow{2}{*}{0,003} \\
\hline & Superior & $\begin{array}{c}51 \\
20,8 \%\end{array}$ & $\begin{array}{c}194 \\
79,2 \%\end{array}$ & $\begin{array}{c}245 \\
100,0 \%\end{array}$ & \\
\hline \multirow{2}{*}{$\begin{array}{l}\text { Doença de fácil contaminação } \\
\text { na ocorrência de acidente } \\
\text { com material biológico }\end{array}$} & Técnico & $\begin{array}{c}195 \\
32,1 \%\end{array}$ & $\begin{array}{c}412 \\
67,9 \%\end{array}$ & $\begin{array}{c}607 \\
100,0 \%\end{array}$ & \multirow{2}{*}{$<0,001$} \\
\hline & Superior & $\begin{array}{c}137 \\
55,9 \%\end{array}$ & $\begin{array}{c}108 \\
44,1 \%\end{array}$ & $\begin{array}{c}245 \\
100,0 \%\end{array}$ & \\
\hline
\end{tabular}

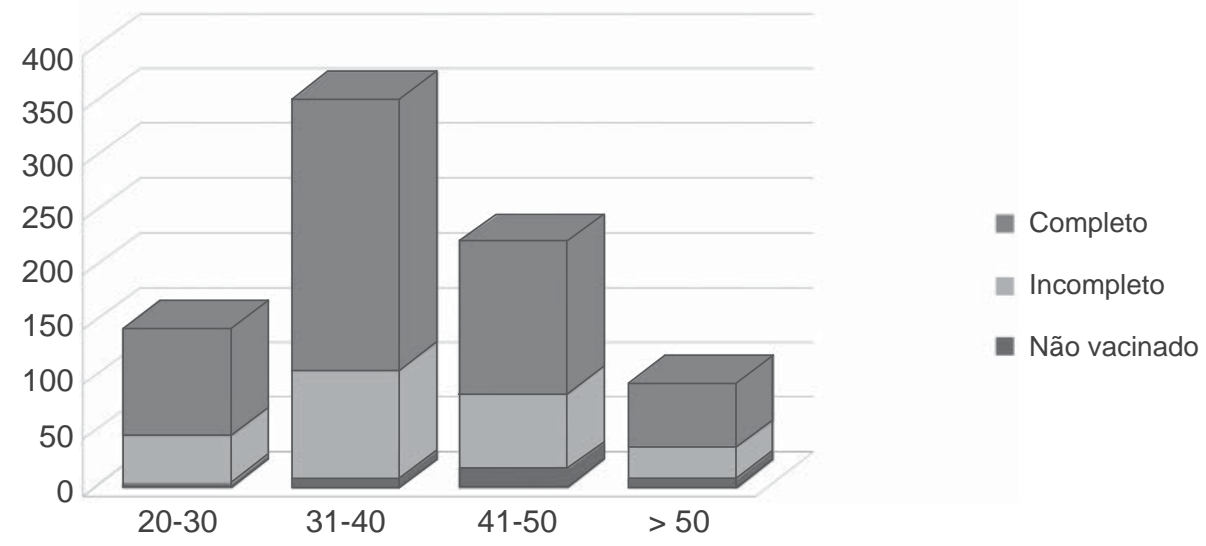

Figura 1 Estado vacinal declarado segundo faixa etária (anos) dos profissionais de saúde de um hospital de emergências de Sergipe, 2007 


\section{Discussão}

Até poucas décadas, o foco da atenção dos profissionais da saúde se limitava ao atendimento e ao cuidado do outro e raramente era valorizada a relação da sua própria saúde com o seu ambiente de trabalho (DA SILVA; CALDEIRA, 2010).

A participação de $63 \%$ de PS do hospital é um indicativo do grau de interesse. Fato relevante quando levado em consideração que o preenchimento dos questionários foi de forma voluntária, a próprio punho e no horário de trabalho na instituição, fatores esses que sabidamente dificultariam a adesão a esse tipo de pesquisa.

Vale ressaltar que, apesar de grande parte $(67,4 \%)$ dos profissionais relatarem ter tido acesso à fonte de informação formal, evidenciou-se principalmente em profissionais do nível técnico, um conhecimento inadequado acerca da presença do vírus da hepatite B no sangue e em fluidos corpóreos, e da forma de transmissão deste vírus no ambiente hospitalar.

Silva et al. (2003) verificaram o conhecimento sobre a transmissão do VHB e o grau de imunização dos profissionais de saúde em um hospital privado da cidade de São Paulo. Esses autores observaram uma relação entre categorias profissionais com maior grau de escolaridade e proporção mais elevada de imunizados, demonstrando uma interferência dos fatores socioculturais na obtenção de conhecimentos referentes a ações de prevenção contra a hepatite. Esses dados corroboram os encontrados no presente estudo a respeito do conhecimento sobre hepatite $\mathrm{B}$, pelos profissionais de saúde, o que reforça a necessidade de constantes investimentos em programas educacionais de orientação relacionados ao tema, tanto em hospitais públicos, quanto nos privados.

A medida mais eficaz contra a hepatite B no ambiente hospitalar é a imunização ativa dos profissionais da saúde (JIA; DING, 2002). Pasternack et al. (1991) comenta em seu estudo que o esquema de vacinação incompleto não é garantia de proteção imunológica e ocasiona desperdício do produto.

No presente estudo, a proporção de imunização declarada pelos profissionais, sem a avaliação da carteira vacinal, foi semelhante aos resultados obtidos no estudo epidemiológico realizado por Almeida e Benatti (2007), no qual as autoras realizaram um levantamento das fichas de notificações de acidentes de trabalho com material perfurocortante, no estado São Paulo. Verificou-se que, dos 379 trabalhadores da saúde acidentados pertencentes às diversas categorias dessa área e de dife- rentes níveis de instrução, 72,8\% estavam com o esquema vacinal completo e $27,2 \%$, incompletos.

Scheneider (1990) e Yoshida (1998) sugerem, em estudos realizados na década passada, a vacinação de todos os estudantes da área da saúde ao ingressarem em cursos técnicos e de graduação no Brasil. Apesar disso, verifica-se que o problema persiste e é preocupante, visto que é observado em diferentes regiões e instituições pesquisadas em todo o mundo, sinalizando a necessidade de estratégias para que se consiga cobertura vacinal próxima de 100\% dos profissionais da saúde (GIR et al., 2008; HATIPOGLU et al., 2007).

De acordo com a cartilha publicada pelo Ministério da Saúde (BRASIL, 2005), a vacina contra o vírus da hepatite B encontra-se disponível no Sistema Único de Saúde para crianças menores de um ano de idade (a partir do nascimento), para crianças e adolescentes entre um e 19 anos e para indivíduos que façam parte dos grupos de risco, como os profissionais de saúde. Nestes, a imunização pode ser realizada em qualquer faixa etária, sendo aplicada em três doses, com intervalo de um mês entre a primeira e a segunda doses, seguida de um período de seis meses entre a primeira e a terceira doses.

Essa política é seguida pela Secretaria de Saúde do Estado de Sergipe. Além disso, é incrementada com a estratégia de dispor a vacina periodicamente em ambientes de grande fluxo de indivíduos, onde equipes de agentes de saúde se estabelecem a fim de realizar a divulgação e a vacinação, principalmente das pessoas que fazem parte dos grupos de risco (PORTAL DA SAÚDE, 2010).

Pode-se verificar que o estado vacinal adequado contra hepatite B está intimamente relacionado aos profissionais com maior tempo de escolaridade, o que pode, possivelmente, ser justificado pelo acesso a informações desde a vida acadêmica sobre as hepatites e suas formas de prevenção. Indica, ainda, a necessidade de maior oferta de capacitações também aos profissionais de nível técnico para maior sensibilização desses à vacinação contra o VHB.

A maioria dos estudos brasileiros foca, em especial, a situação vacinal dos funcionários que compõem a equipe de enfermagem (SANCHES, 2002; XAVIER; SANTOS, 2003), sendo escassa a literatura que aborda outras categorias no ambiente hospitalar. Faz-se mister, então, que pesquisas sobre cobertura vacinal contra hepatite B sejam ampliadas a todos os profissionais que estão envolvidos em atividades com risco de aquisição da doença.

Sabe-se que a vacina contra a hepatite B está disponível gratuitamente desde a década de 1980 a todos os profissionais da saúde (CENTERS FOR DISEASE 
CONTROL, 1987), que tem eficácia comprovada contra a doença (GAZE et al., 2000) e que os benefícios superam os raros riscos de eventos adversos (SCARAUZZI, 2006). Deve-se, portanto, investigar adequadamente os motivos da pequena adesão desses profissionais ao esquema vacinal completo contra a hepatite B.

\section{Conclusão}

A principal fonte de informação adquirida sobre hepatite B relatada pelos profissionais de saúde foi a formal. No entanto, evidenciou-se o conhecimento inadequado, principalmente, entre profissionais de nível técnico, e uma relação estatisticamente significante entre grau de escolaridade e conhecimento correto. Verificou-se uma menor frequência de imunizados contra hepatite $\mathrm{B}$ no nível técnico em relação aos profissionais de saúde de nível superior, de maneira que um terço dos profissionais de saúde apresentavam-se mais susceptíveis ao VHB. Quanto à faixa etária, observou-se que com o aumento da idade, diminuiu significativamente o percentual de indivíduos vacinados.

A hepatite B é sabidamente a doença infecciosa que mais acomete os profissionais de saúde. Porém, observou-se neste estudo o precário conhecimento dos profissionais sobre o tema, uma vez que grande parte dos entrevistados errou os questionamentos que avaliam o nível de conhecimento sobre hepatite B. Essas razões devem motivar os gestores da saúde à implantação de programas de educação continuada, além de pesquisas neste âmbito.

\section{Contribuições de autoria}

Silva, F. J. C. P. da: participou na concepção do estudo, no levantamento de dados e na elaboração do manuscrito. Lima, S. O.: contribuiu no desenho do trabalho, na análise e na interpretação dos dados, na revisão e na aprovação do artigo final. Santos, P. S. F.: colaborou no delineamento do estudo, na análise dos dados, na redação do manuscrito e na aprovação final da versão publicada. Reis, F. do P.: cooperou na interpretação dos dados, na revisão crítica e aprovou o manuscrito final enviado à revista.

\section{Referências}

ALMEIDA, C. A. F.; BENATTI, M. C. C. Exposições ocupacionais por fluídos corpóreos entre trabalhadores da saúde e sua adesão à quimioprofilaxia. Revista $d a$ Escola de Enfermagem - USP, São Paulo, v. 41, n. 1, p. 120-126, 2007.

AZIZ, S. et al. Prevalence of HIV, hepatitis B and $\mathrm{C}$ amonst health workers of Civil Hospital Karachi. Journal Pakistan Medical Association, v. 52, n. 3, p. 92-94, 2002.

BARBOSA, D. B.; SOLER, Z. A. S. G. Afastamentos do trabalho na enfermagem: ocorrências com trabalhadores de um hospital de ensino. Revista Latino-Americana de Enfermagem, v. 11, n. 2, p. 177183, 2003.

BASSO, M. Acidentes ocupacionais com sangue e outros fluídos corpóreos em profissionais de saúde. 1999. 112 f. Dissertação (Mestrado)-Universidade de São Paulo. Escola de Enfermagem, São Paulo, 1999.

BOLYARD, E. A. et al. Guindeline for infection control in healthcare personnel. Infection Control of Hospital Epidemiology, v. 19, n. 6, p. 4007-4063, 1998.

BONANI, P.; BONACCORSI, G. Vaccination aginst hepatitis B in health care workers. Vacine, v. 19, p. 2389-2394, 2001.
BRASIL. Ministério da Saúde. Secretaria de Políticas de Saúde. Coordenação Nacional de DST e AIDS. Manual de condutas: exposição ocupacional a material biológico: hepatite e HIV. Brasília: Ministério da Saúde, 1999.

\section{Ministério da Saúde. Secretaria de}

Vigilância em Saúde. Departamento de Vigilância Epidemiológica. Hepatites virais: o Brasil está atento. Brasília: Ministério da Saúde, 2005.

CANINI, S. R. M. S. et al. Acidentes perfurocortantes entre trabalhadores de enfermagem de um hospital universitário do interior paulista. Revista LatinoAmericana de Enfermagem, v. 10, n. 4, p. 172-178, mar./abr. 2002.

CENTERS FOR DISEASE CONTROL. Update on hepatitis B prevention. Morbidity and Mortality Weekly Report, v. 36, p. 353-366, 1987.

CHIARAKUL, S. Seroprevalence and risk factors of hepatitis B virus infection among health care workers at the Institute of Neurology. Journal of the Medical Association of Thailand, v. 90, n. 8, p. 1536-1545, 2007.

CIORLIA, L. A. S.; ZANETTA, D. M. T. Hepatite C em profissionais da saúde: risco de exposição e infecção. 
Revista Brasileira de Saúde Ocupacional, São Paulo, v. 28, n. 107/108, p. 91-100, 2003.

COUTINHO, A. P. et al. Epidemiologia dos acidentes do material biológico no Instituto de Infectologia Emílio Ribas. In: CONGRESSO BRASILEIRO DE CONTROLE DE INFECÇÃO HOSPITALAR, 8., 2002, Curitiba. Anais... Recife: ABIH, 2002. p. 4-7.

DA SILVA, J. M.; CALDEIRA, A. P. Modelo assistencial e indicadores de qualidade da assistência: percepção dos profissionais da atenção primária à saúde.

Cadernos de Saúde Pública, Rio de Janeiro, v. 26, n. 6, p. 1187-1193, jun. 2010.

GAZE, R. et al. Laboratórios sentinelas - uma proposta para o monitoramento das infecções pelos vírus das hepatites A e B. Informe Epidemiológico do SUS, v. A, n. 1, jan./mar. 2000.

GIR, E. et al. Accidents with biological material and immunization against hepatitis b among students from the health area. Revista Latino-Americana de Enfermagem, v. 16, n. 3, p. 401-406, maio/jun. 2008.

HATIPOGLU, C. A. et al. Vaccination of healthcare workers against hepatitis B virus in a teaching hospital. Journal of Hospital Infection, v. 67, n. 2, p. 200-202, Oct. 2007.

IPPOLITO, G.; PURO, V.; De CARLI, G. The risk of occupational human immunodeficiency virus infection in health care workers. Italian Multicenter Study Group on Occupation Risk of HIV infection. Archives of Internal Medicine, v. 153, n. 12, p. 14511458, 1993.

JIA, H. K.; DING, S. C. Global control of hepatitis B virus infection. The Lancet Infectious Disease, v. 2, n. 7, 2002.

PANILILIO, A. L. et al. Estimate of the annual number of percutaneous injuries among hospital-based healthcare workers in the United States, 1997-1998.

Infection Control of Hospital Epidemiology, v. 25, n. 7, p. 556-562, 2004.

PASTERNACK, J. et al. Imunoprofilaxia da hepatite B, vacinação em um hospital geral de São Paulo com adequação da metodologia utilizada a nossa realidade sócio-econômica. Revista do Hospital das Clínicas da Faculdade de Medicina da USP, v. 46, p. 38-40, 1991.

PERRY, J.; PARKER, G.; JAGGER, J. EPINet report: 2003 percutaneous injury rates. Advanced Exposure Prevention, v. 7, p. 42-45, 2005.

PORTAL DA SAÚDE. Saúde promove ações para lembrar Dia Mundial de Luta contra Hepatites.
Disponível em: <http://www.ses.se.gov.br>. Acesso em: 03 dez. 2010.

RAPPARINI, C. Riscos biológicos e profissionais de saúde. Disponível em: <http://www.riscobiologico. org/riscos/riscos.htm>. Acesso em: 15 dez. 2007.

RISCHITELLI, G. et al. The risk of acquiring hepatitis B or C among public safety workers. American Journal of Preventive Medicine, v. 20, n. 4, p. 299-306, 2001.

SANCHES, F. D. A. Enfermagem do Trabalho: prevenindo a hepatite B em um hospital universitário. 2002. 96 f. Monografia (Residência de Enfermagem do Trabalho)-Hospital Universitário Pedro Ernesto, Universidade do Estado do Rio de Janeiro, Rio de Janeiro, 2002.

SCARAUZZI, D. R. Vacina contra hepatite B. Revista da Associação Médica Brasileira, v. 52, n. 5, p. 288289, 2006.

SCHENEIDER, O. D. Vacinação contra hepatite B no grupo hospitalar Conceição. Mom \&Perspec. Saúde, Porto Alegre, v. 4, n. 1/2, p. 85-90, jun./dez. 1990.

SÊCCO, I. A. O. et al. Acidentes de trabalho em ambiente hospitalar e riscos ocupacionais para os profissionais de enfermagem. Semina: Ciências Biológicas e da Saúde, v. 23, p. 19-24, jan./dez. 2002.

SILVA, R. J. O. et al. Vacinação anti-hepatite B em profissionais de saúde. DST - Jornal Brasileiro de Doenças Sexualmente Transmissíveis, v. 15, n. 3, p. 51-55, 2003.

SOUZA, M. Acidentes ocupacionais e situações de risco para a equipe de enfermagem: um estudo de cinco hospitais do município de São Paulo. 1999. 163 f. Tese (Doutorado)-Universidade Federal de São Paulo. Escola Paulista de Medicina, São Paulo, SP, 1999.

TARANTOLA, A. et al. Assessment of preventive measures for accidental blood exposure in operating theaters: A survey of 20 hospitals in Northern France. American Journal of Infection Control, v. 34, p. 367382, Aug. 2006.

XAVIER, M. S.; SANTOS, R. B. S. A Equipe de enfermagem e os acidentes com material perfurocortante. Enfermagem Brasil, v. 2, n. 1, p. 5-16, jan./fev. 2003.

YOSHIDA, C. F. T. Hepatite B como doença ocupacional. In: TEIXEIRA, P.; VALLE, S. (Orgs.). Biossegurança: uma abordagem multidisciplinar. Rio de Janeiro: Fiocruz, 1998. p. 257-270. 